\title{
Analysis of Black Spot of Traffic Accident in Wuhan Port
}

\author{
Feng Xia ${ }^{1,2, a}$, Ya-dong Yang ${ }^{2}$ \\ ${ }^{1}$ Hubei Provincial Key Laboratory of Inland navigation technique Wuhan 430063, China \\ ${ }^{2}$ School of Navigation, Wuhan University of Technology, Wuhan 430063, China
}

\begin{abstract}
The proposal to build the "Yangtze River Golden Waterway" strategy has enabled the shipbuilding volume of the Yangtze River trunk to grow rapidly. With the increase of ship traffic flow, the Yangtze River water traffic safety is facing severe challenges. This paper draws on the road traffic black spots and existing research methods of water traffic accidents and the definition of water traffic black spots. The DBSCAN algorithm and the accident-level weighted average method are used to establish the blackpoint identification model for waterborne traffic. The model is applied to the Yangtze River mid-stream Wuhan Port 2013-2017 water traffic safety accident as an example. Ten black spots and corresponding boundaries are identified. The black point analysis method used in this paper can effectively quantitatively analyze the spatial distribution and characteristics of water traffic black spots, which can provide a new theoretical basis for ensuring the safety of water traffic.
\end{abstract}

\section{Preface}

Waterway transportation is the main mode of transportation for bulk commodities. It plays an important role in the economic development at home and abroad. The safe navigation of ships has an important impact on the sustainable development of the shipping industry. Frequent waterborne traffic accidents have caused great losses to the country. To a certain extent, it has hindered the sustainable development of the port. In order to ensure the safety of the navigation environment of the ship, it is necessary to analyze and study the black spots of the water traffic in order to provide reference for the water traffic safety management of the maritime authorities and the port dispatching department, and also provide scientific and reasonable guidance for the ship driving personnel.

The research on black spots in water traffic is still in its infancy, and relevant scholars have used fuzzy comprehensive evaluation and hierarchical clustering methods to study the black spots of water traffic accidents. For example, $\mathrm{Xu}$ Yanmin et al[1] used the fuzzy comprehensive evaluation method to evaluate the ship collision risk, and identified the black point of the ship collision accident according to the hazard index identification standard and risk evaluation result of the black spot; Du Shanshan et al.[2] The class algorithm establishes the blackpoint identification model for water traffic, and studies the traffic black spots in the Zhangjiagang section of the Yangtze River. Domestic and foreign scholars have done a lot of research on road traffic black spots[3-7]. Bao Haitao[3] used DBSCANbased analysis method to cluster point sets in space, thus achieving intelligent troubleshooting of traffic accidents.

\footnotetext{
${ }^{\text {a }}$ Corresponding author: 2998609852@qq.com
}

Zhang Man[4] combined with the theory of rough set and inclusion degree, proposed a property selection metric for constructing decision tree-including the importance degree. This method improves the clustering effect well. Michal et al.[5] combined the kernel density estimation with the clustering algorithm to identify black spots and identify the location of road black spots. Liu Anye et al[6] proposed the use of artificial neural network BP algorithm to identify the multiple points of road traffic accidents, enriching the basic theory of traffic accident multiple points identification. Saccomanno et al.[7] first proposed using Poisson regression and empirical Bayesian models to identify black spots in 2001. In 1974 Dunn[8] first proposed the ISODATA algorithm using fuzzy mathematics ( Iterative Self Organizing Data Analysis Techniques Algorithm), by continually modifying the position of the condensation point to classify.

There is a certain similarity between road traffic black spots and water traffic black spots research, which can introduce the related research ideas of road traffic accidents into the study of water traffic accidents[9-10].

Therefore, this paper draws on the definition and identification method of road traffic black spots, based on the existing water accident-prone areas, defines the black spots of water traffic accidents, and proposes a water traffic black spot recognition model based on DBSCAN algorithm; using DBSCAN algorithm to analyze water Traffic accidents, realize reasonable and effective clustering of a large number of water accident data; combine the spatial distribution of water traffic accidents with the accident level, identify the black spots of water traffic accidents and mark the boundaries by studying the black spot identification of water traffic accidents, with a 
view to Quantitative identification of water traffic black spots to prevent only staying in qualitative analysis, in order to obtain more objective and accurate identification results, to provide reference for ship navigation safety.

\section{Definition of water traffic black spots}

The black spot of a traffic accident is the result of the cumulative distribution of traffic accidents within a certain period of time. According to the spatial distribution characteristics of water traffic accidents, the characteristics of accident levels and the definition of road traffic black spots [11-12], water traffic black spots can be defined as: The number or characteristic concentration of water traffic accidents in a certain period of time and specific waters In this case, the water area has an accident level higher than a certain limit. This paper fully considers the spatial distribution of water traffic accidents and the severity of accidents.

\section{Water traffic black dot recognition model}

This paper firstly uses the DBSCAN algorithm to cluster waterborne traffic accidents in space, and then uses the accident level comprehensive weighted average method to determine the blackpoint threshold. As a blackpoint recognition criterion, a waterborne traffic blackpoint recognition model is established. The advantage is that the DBSCAN algorithm is not only suitable for the processing of a large number of accident data, but also is classified by a gradual evolution method, and the method is more scientific; clustering the water traffic accidents from the space, combined with the difference of the accident levels, using the comprehensive weighted average of the accident levels The law distinguishes. The combination of these two methods not only takes into account the location of the accident space, but also the level of the accident.

\subsection{The basic principle of the DBSCAN algorithm}

The basic concept of the DBSCAN algorithm[3] is as follows:

1) Density: The density at any point in the space is the number of points contained in the area where the point is the center of the circle and the radius is $E_{P S}$

2) Neighborhood: The neighborhood of any point in the space is a set of points contained in the area where the point is the center of the circle and the radius of EPS is recorded as $\operatorname{Neps}(p)=\left\{q \in D \mid \operatorname{dist}(p, q) \leqslant E_{P S}\right\}$ Here $D$ is the database.

3) Core point and boundary point: The density of a point in space, if it is greater than or equal to a given threshold Minpts, it is called the core point; otherwise it is called the boundary point.

4) Direct density is reachable: point $p$ is directly accessible from point $q$ and satisfies $p \in \operatorname{Neps}(q)$, $\operatorname{Neps}(q)>$ Minpts.
5) Density up: In database $D$, if there is a set of objects $p_{1}$, $\mathrm{p}_{2} \ldots \mathrm{p}_{\mathrm{n}}$ makes $\mathrm{p}_{1}=\mathrm{q}, \mathrm{p}_{\mathrm{n}}=\mathrm{p}$ for any $\mathrm{p}_{\mathrm{i}} \in \mathrm{D}(1 \leqslant \mathrm{i} \leqslant \mathrm{n}), \mathrm{p}_{\mathrm{i}+1}$ is from $p_{i}$ about EPS and Minpts are directly reachable, so $p$ is called q from EPs.and Minpts.

6) Density connected: There is a point o such that point $p$ and point $\mathrm{q}$ are from o for EPS and Minpts density, then point $\mathrm{p}$ and point $\mathrm{q}$ are connected to EPS and Minpts density.

7) Class: The non-empty set $C$ of the database $D$ is a class, if and only if $\mathrm{p}, \mathrm{q}$, if $\mathrm{p} \in \mathrm{c}$, and from $\mathrm{p}$ density up to $\mathrm{q}$, then $\mathrm{q} \in \mathrm{c} ; \mathrm{p}$, $\mathrm{q}$, if $\mathrm{q} \in \mathrm{c}$, Then $\mathrm{p}$ and $\mathrm{q}$ are density connected.

8) Noise: Points in the database D that do not belong to any class are noise.

\subsection{Implementation of DBSCAN algorithm}

The implementation process of the DBSCAN algorithm is as follows:

1) Initialize the settings.

(1)Add a new attribute ClusterID for storing the clustering result in the original database $\mathrm{D}$, initialize it to zero, and form a new data set $\mathrm{D}_{1}$.

(2) Define TempList to temporarily store the point where the distance between two points is less than the radiuse.

(3) Set the parameters Minpts and EPS.

2) Traverse the set $D_{1}$ and sequentially examine each point as a seed point. $\mathrm{i}=1, \mathrm{j}=1$, and Cluster $=1$.

(1) For any point $p_{i}$, if it's ClusterID $=0$, search for its EPS neighborhood; if $\operatorname{Neps}\left(\mathrm{p}_{\mathrm{i}}\right) \geqslant$ Minpts, then point $\mathrm{p}_{\mathrm{i}}$ is the core point, let it's ClusterID=Cluster, and it's EPS neighborhood All points included are stored in the TempList.

(2) Traverse the TempList and examine each point as a seed point. For any point $\mathrm{q}_{\mathrm{j}}$, if the cluster ID of $\mathrm{q}_{\mathrm{j}}=0$, search for its EPS neighborhood. If $\operatorname{Neps}\left(\mathrm{p}_{\mathrm{j}}\right) \geqslant$ Minpts, then $\mathrm{q}_{j}$ is also a core point. It is the direct density reachable point of point $\mathrm{q}_{\mathrm{j}}$, which belongs to the same class as $p_{i}$, so that it's ClusterID $=$ Cluster, otherwise $q_{j}$ is the boundary point, but it's ClusterID $=$ Cluster. If $\mathrm{q}_{j}$ is the core point, point $\mathrm{o} \in \operatorname{Neps}\left(\mathrm{q}_{\mathrm{j}}\right)$, and o does not belong to TempList, then point o is stored in TempList, and finally point $\mathrm{q}_{\mathrm{j}}$ is deleted from TempList.

(3) $\mathrm{j}=\mathrm{j}+1$, if TempList is not empty, step (2) is performed. 3) $\mathrm{i}=\mathrm{i}+1$, Cluster $=$ Cluster +1 , step (2) is performed until the data set $D_{1}$ is traversed.

4) Delete the search data set TempList.

At this point, the cluster ends, and only the search is needed for the data set to get the final result. The clustering result is recorded in the $\mathrm{D}_{1}$ attribute database, and the point where the field ClusterID $=0$ is a noise point.

\subsection{Water Traffic Black Spot Recognition Based on Accident Level Weighted Average Method}

In combination with the "Water Traffic Accident Statistics Method", the classification of water traffic accidents is used. This paper uses the gradient assignment method commonly used in the study of water traffic safety to convert the severity of the accident into a ratio 
to distinguish the severity of different levels of accidents. Table 1. For the accident class $p_{i}$ obtained by spatial clustering based on the DBSCAN algorithm, a comprehensive weighted method is used to determine the water traffic black spots. First, according to the coefficients in Table 1, the different levels of accidents in the accident class $p_{i}$ are assigned and summed to obtain the blackness value Qi of the accident class $p_{i}$ Secondly, the blackness values $Q_{i}$ of all accidents in the entire jurisdiction are summed. Obtain the black value of the accident in the whole research area $\sum \mathrm{Q}$;

Table1 Coefficient value of the different .grade accident

\begin{tabular}{|c|c|c|}
\hline Serial number & Accident level & Weight \\
\hline 1 & Small accident & 1 \\
\hline 2 & General accident & 2 \\
\hline 3 & Big accident & 3 \\
\hline 4 & Major accident & 4 \\
\hline 5 & Particularly serious accident & 5 \\
\hline
\end{tabular}

Finally, obtain the average value q of all blackness values of the accidents in the study area, and use this value as the judgment threshold of the black spot of the water traffic. If the blackness value of an accident class $p_{i}$ is greater than the average value $\mathrm{Q}$, it can be determined that the accident class $\mathrm{p}_{\mathrm{i}}$ is a water traffic black spot.spot.

$\underset{W_{5}}{Q_{i}=n_{i 1}} \times W_{1}+n_{i 2} \times W_{2}+n_{i 3} \times W_{3}+n_{i 4} \times W_{4}+n_{i 5} \times$

$$
\bar{Q}=\frac{\Sigma Q}{K}
$$

In the formula (1), $\mathrm{Q}_{i}$ is the blackness value of the accident class $p_{i} ; i$ is the accident class number, $i=1,2$, $3, \ldots, \mathrm{k} ; \mathrm{n}_{\mathrm{i} 1}$ is the number of small accidents in the accident class; $\mathrm{n}_{\mathrm{i} 2}$ is the general accident in the accident class $p_{i}$ The number of $n_{i 3}$ is the number of major accidents in the accident class $\mathrm{p}_{\mathrm{i}} ; \mathrm{n}_{\mathrm{i} 4}$ is the number of major accidents in the accident class $p_{i} ; n_{i 5}$ is the number of special accidents in the accident class $\mathrm{p}_{\mathrm{i}} ; \mathrm{W}_{1}, \mathrm{~W}_{2}, \mathrm{~W}_{3}$, $\mathrm{W}_{4}$ and $\mathrm{W}_{5}$ are the weights of the five accident levels in Table 1., and $\mathrm{k}$ in the formula (2) is the total number of accident classes in the whole study area.

\section{Application examples}

\subsection{Overview of Wuhan Port}

Wuhan Port is located in the middle reaches of the Yangtze River, the Zhuankou Port Area (mainly for car rolling), the Zuoling Port Area (mainly for chemical and dangerous goods handling), and the Yangshuo Port Area (mainly for container and bulk cargo handling) Hankou Port Area (mainly for tourist passenger transportation and leisure), Qingshan Port Area (mainly steel and ore), and Yangluo Port Area (mainly container and integrated logistics). Its navigation channels include Hankou Waterway, Wuqiao Waterway, and Qingshanjia Waterway.

From 2003 to 2017, there were 434 water traffic accidents in Wuhan Port, including 393 small accidents and 41 minor accidents. According to the statistics of the maritime affairs department, the above-mentioned 434 accidents occurred in the waters of Hankou Waterway, Wuqiao Waterway, Qingshan Clip Waterway, Tianxing Island Anchorage, and liquid cargo ship waiting for anchorage. Use Acr GIS visualization and other functions to assist in the spatial distribution of accidents. By importing the accident location coordinates, you can display the spatial distribution of the water accident on the map.

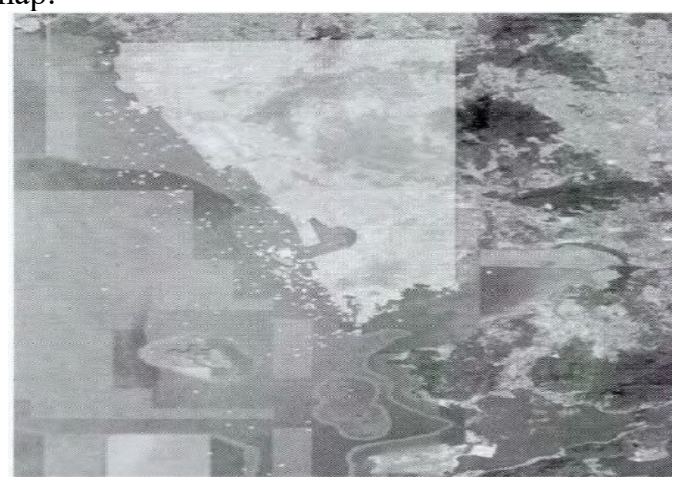

Fig.1 Real scene distribution of water accident in wuhan

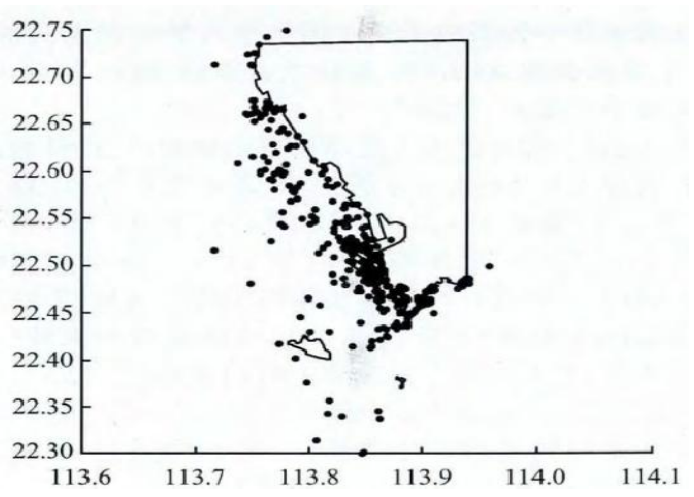

Fig.2 Distribution of water traffic accident in wuhan port

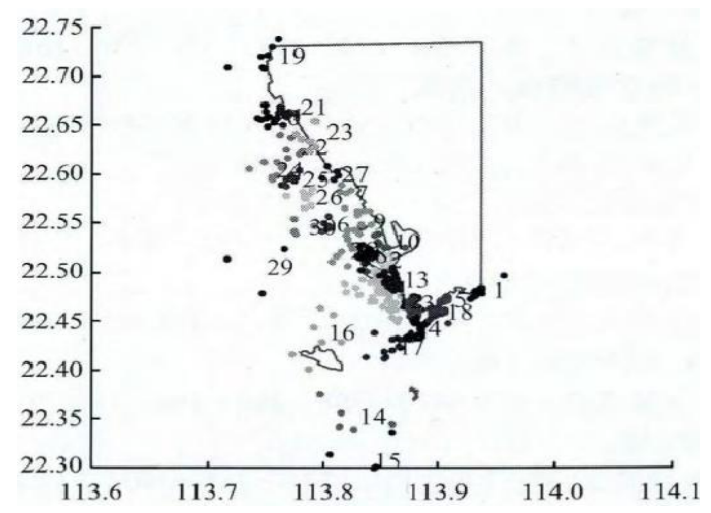

Fig.3 Clustering resuits of waterway traffic accident with 30 clusters 


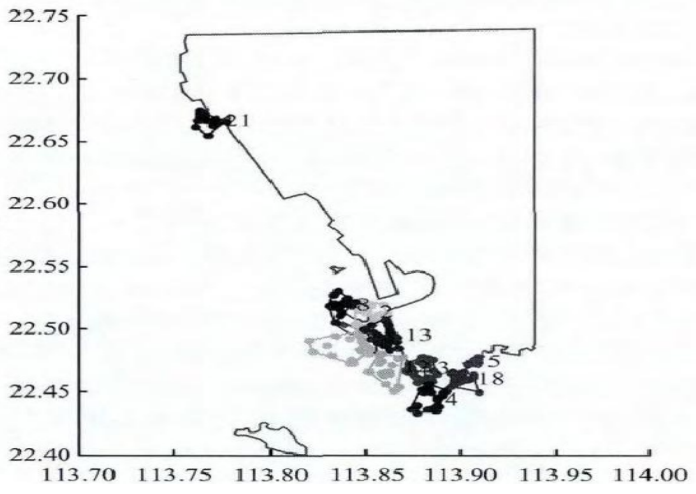

Fig.4 Position of 10 waterway traffic accident black-spots

The distribution of accidents in Wuhan Port is shown in Figures 1 and 2.

\subsection{Identification of waterborne black spots in Wuhan Port waters}

First, construct a geographic coordinate data matrix D of the accident in the study area. Secondly, using ArcGIS to import the coordinates of the accident location, the spatial distribution of the complete water accident can be displayed, as shown in Figure 2. Finally, the initial values of the control parameters EPS and Minpts are selected, and the accident clustering is obtained through the intelligent DBSCAN algorithm. This paper refers to a new method to determine EPs and Minpts adaptively by analyzing the statistical characteristics of data sets. This method is based on DBSCAN algorithm and proposes a new method to determine parameters EPS $_{P S}$ and Minpts adaptively based on distance, which is called I-DBSCAN. The main idea of this method is to select the Eps value and Minpts value according to the statistical characteristics of the data set itself, which is finally determined as Eps $=5 \mathrm{~km}$ and Minpts $=10$ by this method[13-14]. and 434 water traffic accidents in the study area are classified into 30 categories, as shown in Figure 4. According to equations (1) and (2), the blackness value and the accident blackness judgment threshold of each accident type are calculated, as shown in Table 2.

Table2 Risk degree of each accident cluster and threshold value of the accident black-spot

\begin{tabular}{|c|c|c|c|}
\hline Clustering & Blackness value & Clustering & Blackness value \\
\hline 1 & 10 & 16 & 8 \\
\hline 2 & 51 & 17 & 13 \\
\hline 3 & 42 & 18 & 23 \\
\hline 4 & 28 & 19 & 10 \\
\hline 5 & 21 & 20 & 10 \\
\hline 6 & 5 & 21 & 27 \\
\hline 7 & 5 & 22 & 7 \\
\hline 8 & 26 & 23 & 11 \\
\hline 9 & 15 & 24 & 10 \\
\hline 10 & 13 & 25 & 11 \\
\hline 11 & 31 & 26 & 9 \\
\hline 12 & 23 & 27 & 8 \\
\hline 13 & 53 & 28 & 8 \\
\hline 14 & 5 & 29 & 6 \\
\hline 15 & 6 & 30 & 8 \\
\hline \multicolumn{5}{|c|}{ Threshold: 16.77} \\
\hline
\end{tabular}

Comparing the blackness value of each accident category and the blackout point threshold of the accident in Table 3, it can be seen that there are 10 accidental blackness values greater than the blackpoint threshold of the accident, so the 10 locations are determined as black spots of water traffic accidents, as shown in Figure 5. The 10 identified water traffic black spots are marked from the outside (in the order of extension from the river to the shore), which are 1 (category 4), 2 (category 18), 3 (category 5), 4 (Category 3), 5 (Category 12), 6 (Category 11), 7 (Category 13), 8 (Category 2), 9 (Category 8), 10 (Category 21), respectively Corresponding to Hankou waters (No. 1 black spot), Hankou waters (No. 2 black spot), Hankou waters (No. 3 black spot), Tianxing Island anchorage (No. 4 black spot), Qingshan clip water area (No. 5 black spot), Qingshan Clip waters (black spot 6), Qingshan clip waters (black spot 7), tanker waiting for anchorage (black point 8), Wuqiao waters (black point 9), Wuqiao waters (black point 10) The boundary range of these 10 black points ( the boundary accident point number) is shown in Figure 5 , respectively.

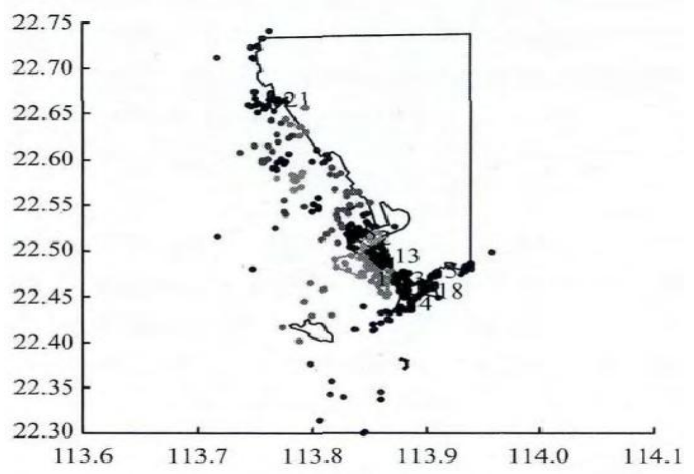

Fig.5 Boundary of 10 waterway traffic accident blackspots in wuhan port

Black spot No. 1: The area enclosed by the line of 103374-255-244-375-373-103.

Black spot No. 2: The area enclosed by the line of 28225-287-284-127-28.

Black spot No. 3: The area enclosed by the line of 31292-69-90-222-136-294-31.

Black spot No. 4: The area enclosed by the line of 6-58256-233-338-416-295-101-148-106-6.

Black spot No. 5: The area enclosed by the line of $12-$ 163-381-56-164-12.

Black spot No. 6: The area enclosed by the line of $80-$ 137-419-86-223-179-224-102-80.

Black spot No. 7: The area enclosed by the line of 20327-158-321-182-411-313-226-414-20.

Black spot No. 8: The area enclosed by the line of 9-227401-354-355-300-275-274-213-9.

Black spot No. 9: The area enclosed by the line of 7-176420-368-394-196-112-7.

Black spot No. 10: The area enclosed by the line of $37-$ 149-151-237-345-140-37.

According to the blackness value of each black point, the black point risk degree is from the highest to the lowest, 
7th black point, 8th black point, 4th black point, 6th black point, 1st black point, 10th black point. , 9th black dot, 5 th black dot, 2nd black dot, 3rd black dot.

The location identified by the maritime traffic black-spot recognition model is consistent with the maritime department's statistically concentrated multiple locations, and the location of the black-spot recognition results is more specific and the boundaries are more clear, which is conducive to water traffic supervision.

\section{Conclusion}

1) A black spot recognition model for water traffic is established. Using the DBSCAN algorithm and the accident level weighting method, the water traffic black spots are studied from the spatial distribution with the number of accidents and the accident level as indicators.

2) Based on the built model, a case study of Wuhan Port 2013-2017 traffic accident is carried out to identify the traffic black spots and corresponding black point boundaries of the water area, and determine the severity of each black point, which is consistent with the accident occurrence position. And the boundaries are clearer.

3) In the follow-up study, the research on the causes of black spots in water traffic accidents will be carried out based on the identified black spots of water traffic accidents.

\section{References}

1. XU Yanmin , TANG Chengang . Identifying gloomy spots of ship collision accident with grid theory [J] . Navigation of China, 2013, 36(4):72-75.

2. DU Shanshan, CHEN Houzhong Identification of waterway traffic black-spot [ J J Journal of Dalian Maritime University, 2016, 42(2):46-5.

3. BAO Haitao. Evaluation of accident black spots on roads using clustering algorithm [D ] Changchun: Jilin University, 2005.

4. ZHANG Man . Research on clustering and classification algorithm based on rough set and inclusion degree $[\mathrm{D}]$. Qingdao: Qingdao Technological University, 2015.
5. MICHAL B , RICHARD A , ZBYNEK J . Identification of hazardous road locations of traffic accidents by means of kernel density estimation and cluster significance evaluation $[\mathrm{J}]$. Elsevier, 2013, 55( 3) :265.

6. SACCOMANNO F, GROSSIR, GRECO D, et al . Identifying blackspots along highway SS107 in southern Italy using two models $[\mathrm{J}]$. Journal of Transportation Engineering. 2001, 127(6) : 515-522.

7. DUNN J C . A fuzzy relative of the ISODATA process its use in delecting compact well-separated clusters $[\mathrm{J}]$. Journal of Cybenetics, 1974, 3(3) : 3257.

8. XIAO Shen, GUO Xiucheng, XU Jiandong . Application fuzzy clustering method in the highway traffic black-spot cause of formation analysis $[\mathrm{J}]$. Journal of Transportation Systems Engineering and Information Technology, 2002, 2(3) : 40-43.

9. WANG Hai . Based on spatial analysis technology of traffic accident black point identification and cause analysis [D] . Beijing: Tsinghua University, 2014.

10. SANDHU H A, SINGH G, SISODIA M S, et al. Identification of black spots on highway with kernel density estimation method [ J ] . Journal of the Indian Society of R emote Sensing, 2016, 44 (3) :457-464 . 11. GEURTS K, WETS G, BRIJS $\mathrm{T}$, et al . Identification and ranking of black spots: sensitivity analysis [ J ] . Transportation Research Record Journal of the Transportation Research Board, 2004, 1897(1) : 34-42.

12. ANDREAS G, KYRIACOS C M. Black spots identification through a Bayesian networks quantification of accident risk index $[\mathrm{J}]$. Elsevier, 2013, 28(3) : $28-43$.

13. Jiang Hua, Li Jing, Yi Shenghe, et al . A new hybrid meth — od based onpartitioning — based DBSCAN and antclustering $[\mathrm{J}]$ Expen Systems with Applications , 2011, 38(8):9373-9381.

14. Hong FangZhou,Peng Wang. DBSCAN algorithm for parameter adaptive determination method $[\mathrm{J}]$. Journal of xi 'an university of technology,2012,28(3):289-292. DOI:10.3969/j.issn.1006-4710.2012.03.007. 